Article original

\title{
Impact of the nature of anticoagulant on the determination of gamma glutamyl transferase activity in blood
}

\author{
Impact des anticoagulants sur la détermination de l'activité de la gamma glu- \\ tamyl transférase dans le sang
}

\author{
Mustapha ZENDJABIL ${ }^{(1,2)}$, Ismahene RIKAI ${ }^{(1,3)}$, Ghizlene BENHAMED ${ }^{(1,3)}$ \\ 'Département de Pharmacie. Faculté de Médecine d'Oran, Algérie. \\ 'Service de Biochimie. Centre Hospitalier Universitaire d'Oran, Boulevard Docteur Benzerdjeb, Oran, Algérie. \\ 3Laboratoire de Front de Mer d'Oran. Rue Sahraoui Meknous, Oran, Algérie.
}

Auteur correspondant: zendjabil.mustapha@univ-oran1.dz

Article reçu le 12/09/2018/Accepté le 19/11/2018

\section{KEY WORDS \\ Gamma glutamyl trans- ferase ; pre-analytical variables; influence of anticoagulants ; Lithium heparinate.}

\begin{abstract}
Background - Gamma-glutamyl transferase (GGT) is routinely used as a highly sensitive marker of hepatobiliary disease, alcohol consumption and more recently as predictive biomarker of cardiovascular events. In current practice, laboratories measure the GGT activity on tube containing lithium heparin. Except that there is no evidence that GGT activity can be reliably determined with this anticoagulant. The purpose of this study is to evaluate the impact of the anticoagulant on the determination the GGT activity in blood samples.

Methods - Blood samples of 30 patients were collected in three types of tubes: dry tube, tube containing lithium heparinate and another containing dipotassium ethylene diamine tetraacetate (K2-EDTA). The statistical analysis includes correlation studies, Bland Altman's analysis, as well as the comparison of biases to analytical change limit (ACL) and reference change value (RCV).

Results - Results obtained on heparinized plasma are globally overestimated comparatively to the serum $(p=0.001)$. Whereas, the results obtained on EDTA plasma are more comparable to those of serum $(\mathrm{p}=0.356)$. Taking the dry tube as a reference, the calculated bias for heparinized plasma $(+49.55 \%)$ samples is larger than the ACL (5.24\%) and the RCV (37.52\%). For EDTA plasma samples, this bias (-13.40\%) is lower than RCV, but higher than ACL.

Conclusion - Our results show that the determination of GGT activity must be done on serum samples. If ever plasma should be used, we recommend collecting specimens on EDTA anticoagulant.
\end{abstract}




\section{MOTS CLÉS}

Gamma glutamyl transférase; variables pré-analytiques; influence des anticoagulants; héparinate de Lithium.

\section{Introduction}

The control of pre-analytical variables is crucial to ensure quality of laboratory testing. In fact, errors that occur during this phase can represent $75 \%$ of all errors encountered in the laboratory [1]; $26 \%$ of them can have adverse effects on the patient's management such as the prescription of unnecessary examinations or increase in the length of hospitalization. On the one hand, due to advances in automation, and on the other hand, to quality control assessment, errors in the analytical phase have become very low [2]. However, the quality control serum does not allow the evaluation of pre-analytical variables and in current practice, laboratories measure the parameters of general biochemistry on a single tube containing lithium heparin. This simplifies the organization of the laboratory. Except that, there is no evidence that each of these parameters can be reliably determined with this anticoagulant.

Gamma-glutamyl transferase (GGT) (EC. 2.3.2.2) catalyzes the first step in the degradation of extracellular glutathione (GSH), allowing for the precursor amino acids to be used for intracellular GSH synthesis. Moreover, GGT favors the cellu- lar supply of GSH, which, is an important antioxidant of the cell [3]. The GGT is especially active in the liver, proximal renal tubule, pancreas, and intestine, but most of the circulating activity in healthy individuals is of hepatic origin [4]. So, the GGT is routinely used as a highly sensitive marker of hepatobiliary disease and alcohol consumption [5]. More recently, the GGT was described as an independent cardiovascular risk factor and could serve as a predictive biomarker for cardiovascular events such as stroke risk $[6,7]$. The purpose of this study is to evaluate the impact of anticoagulant on the determination of GGT activity in blood samples.

\section{Material and methods}

\section{Blood collection}

The study was conducted at the laboratory of Front de Mer in Oran (Algeria) during April 2018. We randomly selected samples from thirteen patients. For each of these patients, blood samples were collected in three types of tubes, according to the nature of the anticoagulant: dry tube, lithium 
heparinate tube and dipotassium ethylene diamine tetraacetate (K2-EDTA) tube. For obvious ethical reasons, we selected samples from patients who had to have blood samples collected on all three types of tubes for performing other biological tests requested by attending physicians. So no additional samples were taken. Lipemic, hemolysed or icteric samples were excluded from our research. The blood specimens were collected on fasting subjects at the morning between 8:00 am and 9:30 am by the same sampler. Then, the samples are centrifuged at $2700 \mathrm{~g} / \mathrm{min}$ for $10 \mathrm{~min}$ on the centrifuge Rotofix $32 \mathrm{~A}$ Hettich $₫$, with a delay of one hour and half for the dry tubes without gel separator. Whereas, the centrifugation of heparinized and K2-EDTA tubes is carried out within one hour of sampling.

\section{Instrumentation and GGT activity measurement}

The Determination of GGT activity was performed on the clinical chemistry analyzer HumaStar $80 \AA$ (Human Diagnostics ${ }^{\circ}$, Germany). We use Biosystem $®$ reagents; the principle of the method is as follows: GGT catalyzes the transfer of the $\gamma$-glutamyl group from $\gamma$-glutamyl-3- carboxy-4-nitroanilide to glycylglycine, liberating 3-carboxy-4-nitroaniline. The catalytic concentration is determined from the rate of 3-carboxy-4-nitroaniline formation at $405 \mathrm{~nm}$ in kinetic mode [8].

Y-Glutamyl-3-carboxy-4- nitroanilide + Glycylglycine $\longrightarrow$ Y-Glutamyl-glycylglycine +3-carboxy-4nitroaniline Calibrations and quality control are carried out according to the supplier's recommendations.

\section{Statistical analysis}

We studied the correlation between the measurements obtained for the three types of tubes, as well as realize a Bland-Altman analysis. Then, the bias is calculated according to the following formula: Bias $(\%)=100$ * (the average - the reference average) / the reference average. The serum was taken as reference.

The bias obtained was compared to analytical change limit $(A C L)$. The $A C L$ represents the maximum analytic variation makes it possible to interpret a measured difference based on the coefficients of variation ( $\mathrm{CVa}$ ), using the following formula: $\mathrm{ACL}=1.96 \times \sqrt{2} \times \mathrm{CVa}$ [9]. The CVa was calculated from the quality control values collected over a six-month period. The term « $1.96 »$ represents the standard deviation corresponding to the level of statistical significance for a bidirectional change $(1.96=95 \%)$ and $\sqrt{2}$ denotes the probability of a bidirectional change.

In addition, we compared the bias to the reference change value (RCV), which incorporates the acceptable inaccuracy based on the intra-individual biological variation and the laboratory-related factors (analytical variables) [10]. This value appears as the maximum biological variation that can be tolerated. It is calculated according to the following formula: $\mathrm{RCV}=1,96 \times \sqrt{ } 2 \times 5 \mathrm{CVa} 2+\mathrm{CVw} 2[11,12]$. The CVw represents the intra-individual variability that was derived from the enumeration of biological variation for 316 analytes by Ricos et al. [13]. The difference will be considered significant if the absolute value of the average bias exceeds the calculated limits of the ACL and the RCV.

Statistical treatments were carried out using Excel $₫$ Spreadsheet while Bland-Altman analysis and t-test was realized using XLSTAT $®$ software.

\section{Results}

The correlation between the results obtained in serum to those obtained in heparinized plasma provides a slope line a $=1.027$ and $y$ intercept $b=-11.27$ with a correlation coefficient $r=0.825$ which reflects a good correlation between the two variables (Fig. 01). Moreover, the correlation between the results obtained in serum to those obtained in EDTA plasma provides a straight line of slope $a=0.989$, y intercept $b=3.007$ and a correlation coefficient $r=0.861$ which also reflects a good correlation between the two variables.

Fig 1 : Graphical representation of correlation lines of the experimental results obtained for heparin and EDTA tubes comparing to drain tube.
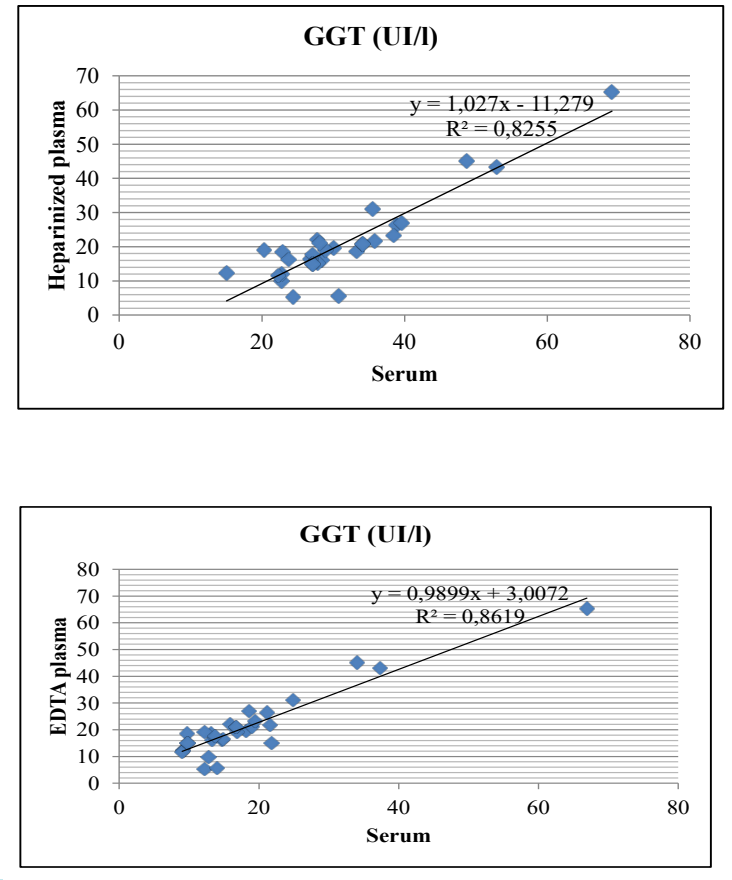
Bland Altman's analysis (Figure 02 and Figure 03) shows that the results obtained on heparinized plasma are globally overestimated relatively to the serum. Whereas, the results obtained on EDTA plasma are comparable to those of serum with the exception of three values that are out of the limits.

Figure 2: Graphical representation of the results of Bland-Altman analysis obtained for GGT activity (The ordinate shows the difference between the value measured on heparinized plasma $(Y)$ and the value measured on the serum $(X)$. The abscissa shows the average of the measurements).

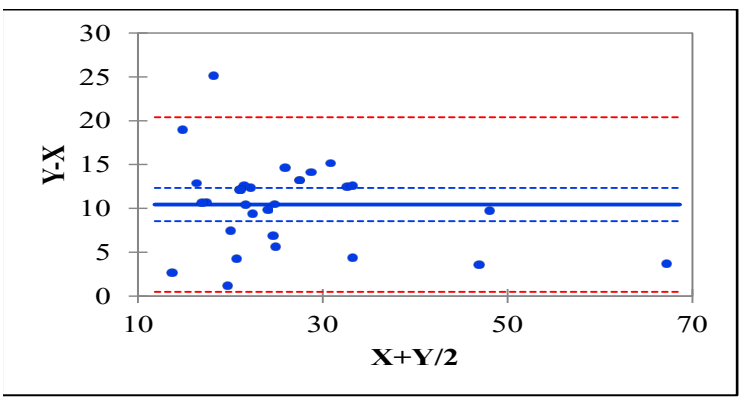

Figure 3: Graphical representation of the results of Bland-Altman analysis obtained for GGT activity (The ordinate shows the difference between the value measured on EDTA plasma $(\mathrm{Y})$ and the value measured on the serum $(X)$. The abscissa shows the average of the measurements).

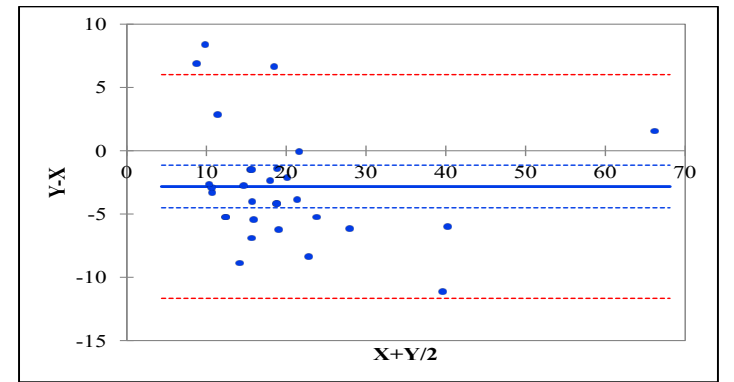

The t-test confirms these observations (Table 1 and Table 2 ) with a significant difference $(p=0.001)$ between the determination of the GGT activity on lithium heparinate tubes compared to the dry tubes.

While, there is no significant difference with the tubes containing EDTA ( $p=0.356)$.
Table 1: Experimental results obtained for the three types of samples.

\begin{tabular}{|c|c|c|c|c|c|}
\hline Tube & $\begin{array}{l}\text { Number of } \\
\text { samples (n) }\end{array}$ & $\begin{array}{c}\text { Minimal value } \\
(\mathrm{U} / / /)\end{array}$ & $\begin{array}{c}\text { Maximal value } \\
\text { (UI/I) }\end{array}$ & Mean (U//I) & $\begin{array}{c}\text { Standard } \\
\text { deviation } \\
(U / / /)\end{array}$ \\
\hline $\begin{array}{l}\text { Plain tube : no } \\
\text { anticoagulant }\end{array}$ & \multirow{3}{*}{30} & 5.34 & 65.37 & 21.05 & 12.13 \\
\hline $\begin{array}{l}\text { Lithium } \\
\text { Heparin } \\
\text { anticoagulant }\end{array}$ & & 15.03 & 69.06 & 31.48 & 10.74 \\
\hline \begin{tabular}{|l|} 
EDTA \\
anticoagulant
\end{tabular} & & 9.00 & 66.93 & 18.23 & 11.38 \\
\hline
\end{tabular}

Table 2: Comparison between the GGT activity results obtained on heparinized plasma and EDTA compared to serum.

\begin{tabular}{|c|c|c|c|c|c|c|}
\hline Comparison & Bias (\%) & p-value & CVa (\%) & CVW (\%) & ACL (\%) & RCV (\%) \\
\cline { 1 - 5 } $\begin{array}{c}\text { Heparin Vs } \\
\text { plain tube }\end{array}$ & +49.55 & 0.001 & 1.921 & 13.40 & 5.24 & 37.52 \\
\cline { 1 - 4 } $\begin{array}{c}\text { EDTA vs } \\
\text { plain tube }\end{array}$ & -13.40 & 0.356 & & & \\
\hline
\end{tabular}

Taking the dry tube as a reference, the calculated bias for heparinized plasma samples is larger than the $A C L$ and the RCV. For EDTA plasma samples, this bias is lower than the RCV, but higher than the ACL.

\section{Discussion}

GGT is an enzyme commonly measured in medical analysis laboratories. However, there are no studies that suggest if other tubes than the dry tube can be used. In addition, the recommendations of suppliers found on the flyers of reagents are different. Thus, for the same assay method, some suppliers advocate the use of serum only. While others mention the possibility of using heparinized plasma. For example, in the Biosystem $®$ reagent prospectus, it has mentioned the possibility of measuring GGT in the three types of samples interchangeably. Conversely, Pointe Scientific $\circledast$ reagents recommend the serum only and Beckman Coulter ${ }^{\circledR}$ recommends serum samples and the plasma collected on EDTA tube.

The results of this study show that the collection of blood samples should not be done with tubes containing lithium heparin which leads to an overestimation of the results. This interference with heparin can be explained by turbi- 
Impact of the nature of anticoagulant on the determination of gamma glutamyl transferase activity in blood

dity production in the reaction mixture [14]. The comparison between the determination of GGT activity in Wistar rat serum, heparinized plasma and serum supplemented with heparin showed GGT activities of $45.1 \pm 9.5 \mathrm{U} / \mathrm{l}$ (mean $\pm \mathrm{SD}), 161.2 \pm 46.1 \mathrm{U} / \mathrm{l}$, and $93.3 \pm 30.9 \mathrm{U} / \mathrm{l}$ respectively [15]. In human samples, very little data exist with conflicting results; some report show an overestimate of results [16], while others find an underestimate [17]. In addition, these studies use older analyses with lower sample number.

The results obtained on EDTA assay are more comparable to those obtained on serum except that the bias is higher than the RCV. Therefore, we recommend excluding this anticoagulant except some emergencies situations, where we take a single tube for others tests as blood count formula to offer a better patient care. Other types of anticoagulants are not evaluated in this study because citrate, oxalate, and fluoride are known to give GGT values 15\% lower than those obtained from serum [18]. On the other hand, it would be interesting to evaluate the influence of separator gels on the determination of GGT activity.

\section{Conclusion}

To our knowledge, this is the first study to investigate the influence of the nature of anticoagulant on the determination of GGT activity in human blood samples. The GGT activity determination must be done on serum samples. If ever plasma should be used, we recommend collecting specimens on EDTA anticoagulant. Note, however, that it would be interesting to verify these results on a larger number of samples with more pathological values. In addition, we advise the biologists to be vigilant by checking also the influence of the anticoagulant on the results of other biochemical parameters in order to satisfy the requirements of the norm ISO 15189.

\section{Conflicts of interest : None.}

\section{References}

[1]. Green SF. The cost of poor blood specimen quality and errors in preanalytical processes. Clin Biochem 2013;46(13-14):175-9.

[2]. Lippi G, Becan-McBride K, Behúlová D, Bowen RA, Church S, Delanghe $\mathrm{J}$ et al. Preanalytical quality improvement: in quality we trust. Clin Chem Lab Med 2013;51(1):229-41.

[3]. Faiq M, Shafi A, Khalil F, Ayub A, Saeed A. To Find out the Association of Mortality in Acute Heart Failure patients with Raised Serum Gamma-Glutamyl Transferase (GGT). Pak J Med Sci 2018;12(1):121-2.
[4]. Jiang S, Jiang D, Tao Y. Role of gamma-glutamyltransferase in cardiovascular diseases. Exp Clin Card 2013;18(1):53.

[5]. Ndrepepa G, Xhepa E, Colleran R, Braun S, Cassese S, Fusaro M et al. Gamma-glutamyl transferase and atrial fibrillation in patients with coronary artery disease. Clin Chim Acta 2017;465:17-21.

[6]. Yang W, Kim CK, Kim DY, Jeong HG, Lee SH. Gamma-glutamyl transferase predicts future stroke: A Korean nationwide study. Ann Neurol 2018;83(2):375-86.

[7]. Ndrepepa G, Holdenrieder S, Cassese S, Fusaro M, Xhepa E, Laugwitz KL et al. A comparison of gamma-glutamyl transferase and alkaline phosphatase as prognostic markers in patients with coronary heart disease. Nutr Metab Cardiovasc Dis 2018;28(1):64-70.

[8]. IFCC Primary reference Procedures for the measurement of catalytic activity concentrations of enzymes at $37^{\circ} \mathrm{C}$. Part 6 . Reference procedure for the measurement of catalytic concentration -Glutamyltransferase. Clin Chem Lab Med 2002;40:734-8.

[9]. Monneret D, Corlouer C, Bigot J, Atlan G, Alkouri R, Mestari F et al. Comparison of a 10-vs. 15-min centrifugation time for chemical and immunochemical assays and impact on turnaround time in a hospital laboratory. Clin Chem Lab Med 2016;54(4):e117-e121.

[10]. CDromigny JA, Robert, E. (2017). Stabilité du potassium sanguin: influence du délai, de la température et du transport sur les dosages sériques et plasmatiques durant 10 heures de conservation. Ann Biol Clin 2017;5(4):369-74.

[11]. Monneret D, Godmer A, Le Guen R, Bravetti C, Emeraud C, Marteau A et al. Stability of Routine Biochemical Analytes in Whole Blood and Plasma From Lithium Heparin Gel Tubes During 6-hr Storage. J Clin Labo Anal 2016;30(5):602-9.

[12]. Dupuy AM, Cristol JP, Vincent B, Bargnoux AS, Mendes M, Philibert $P$ et al. (2018). Stability of routine biochemical analytes in whole blood and plasma/serum: focus on potassium stability from lithium heparin. Clin Chem Lab Med 2018;56(3):413-21.

[13]. Ricos C, Alvarez V, Cava F, García-Lario JV, Hernández A, JiménezCV, et al. Current databases on biological variation : pros, cons and progress. Scand J Clin Lab Invest 1999;59:491-552, Accès en avril 2018 http://www.westgard.com/biodatabase1.htm.

[14]. Panteghini M, Bais R. Serum enzymes in Tietz textbook of clinical chemistry and molecular diagnostics-e-book. Elsevier Health Sciences, sixth edition, 2017:421.

15]. Castro-e-Silva OJ, Franco CF, Picinato MA, Souza ME, Mazzetto $S A$, Ceneviva R. Heparin-induced increase in plasma and serum gamma-glutamyl transpeptidase activity. Braz J Med Biol Res 1989;22(11):1333-5.

[16]. Harr R, Bond L, Trumbull D. A comparison of results for serum versus heparinized plasma for 30 common analytes. Lab Med 1987;18(7):449-55.

[17]. Weber JA. Elimination of heparin interference in the determination of gamma-GT. Clin Chim Acta 1987;169(2-3) :345-6.

[18]. Sepulveda J. Challenges in Routine Clinical Chemistry Analysis: Proteins and Enzymes. In Accurate Results in the Clinical Laboratory, 2013:131-48. 
